\title{
The inflammasome in leprosy skin lesions: an immunohistochemical evaluation
}

This article was published in the following Dove Press journal: Infection and Drug Resistance

\author{
Luciana Mota Silva' \\ Jorge Rodrigues de Sousa ${ }^{2,3}$ \\ Kelly Emi Hirai' \\ Leônidas Braga Dias Jr' \\ Ismari Perini Furlaneto' \\ Francisca Regina Oliveira \\ Carneiro' \\ Tinara Leila de Souza \\ Aarão' \\ Mirian Nacagami Sotto 4,5 \\ Juarez Antonio Simões \\ Quaresma ${ }^{1-3,5}$ \\ 'Center of Biological and Health \\ Science, State University of Para, \\ Belem, Brazil; ${ }^{2}$ Tropical Medicine \\ Center, Federal Do Para University, \\ Belem, Brazil; ${ }^{3}$ Evandro Chagas \\ Institute, Ministry of Health, \\ Ananindeua, Brazil; ${ }^{4}$ School of \\ Medicine, Sao Paulo University, Sao \\ Paulo, Brazil; ${ }^{5}$ Tropical Medicine \\ Institute, Sao Paulo University, Sao \\ Paulo, Brazi
}

Correspondence: Juarez Antonio Simões Quaresma

Tropical Medicine Center, Federal Do Para University, Av. Generalíssimo

Deodoro 92, Umarizal, Belem, PA 66055-

240, Brazil

Tel +559132010966

Email juarez.quaresma@gmail.com
Objective: Leprosy is a chronic infectious disease presenting with a spectrum of clinical manifestations that correspond to the type of immune response that develops in the host. Factors that may be involved in this process include inflammasomes, cytosolic proteins responsible for the activation of caspase 1, IL-1 $\beta$ and IL-18 secretion, and induction of a type of death called pyroptosis.

Patients and methods: We evaluated the expression of inflammasome markers (nucleotidebinding oligomerization domain-like receptor containing pyrin domain 1 [NLRP1], nucleotidebinding oligomerization domain-like receptor containing pyrin domain 3 [NLRP3], caspase 1, IL-1 $\beta$, and IL-18) by immunohistochemistry in 43 samples of skin lesions of leprosy patients from the groups indeterminate (I) leprosy (13 patients), tuberculoid (TT) leprosy (15 patients), and lepromatous leprosy (LL; 15 patients).

Results: The evaluated markers were most upregulated in LL lesions, followed by lesions of TT leprosy and I leprosy. Differences were statistically significant between the I leprosy and LL leprosy forms and between the I leprosy and TT leprosy forms. Positive and significant correlations were found between IL-18 and caspase 1 in LL ( $r=0.7516, P=0.0012)$ and TT leprosy $(r=0.7366, P=0.0017)$. In I leprosy, correlations were detected between caspase 1 and IL-1 $\beta$ $(r=0.6412, P=0.0182)$, NLRP1 and IL-18 $(r=0.5585, P=0.473)$, NLRP3 and IL-18 $(r=0.6873$, $P=0.0094)$, and NLRP1 and NLRP3 ( $r=0.8040, P=0.0009)$.

Conclusion: The expression of inflammasome markers in LL lesions indicates the ineffectiveness of this protein complex in controlling the infection. Caspase 1 may be involved in the pyroptotic cell death in the lepromatous form of the disease. Inflammasomes may act together in the initial phase of I leprosy; this phenomenon may influence the clinical outcome of the disease.

Keywords: IL-1 $\beta$, NLRP, Mycobacterium leprae, immune response, pyroptosis

\section{Introduction}

Leprosy is a chronic infectious disease caused by Mycobacterium leprae. The disease evolves with characteristic granulomatous lesions manifested mainly in the skin and peripheral nerves. ${ }^{1}$ The clinical and histopathological characteristics of the disease have a spectrum that depends on the pattern of the host immune response; thus, the disease develops into a polarized form called tuberculoid (TT) leprosy, which is related to the T helper 1 (Th1) cytokine profile (mainly IL-2 and IL-12), and lepromatous leprosy (LL) form, which is related to the Th2 cytokine profile (mainly IL-4). Between these clinical patterns, there is borderline leprosy that shows cytokine expression that varies with the immune phenotype of the cells. ${ }^{2}$ Indeterminate (I) leprosy is the initial 
clinical phase of the disease. In this phase, the bacillus cells generally interact with the immune system, determining the later evolution of the infection. ${ }^{3}$

Recently, other factors associated with innate immunity, such as macrophages and new cytokine profiles, have been implicated in the immunopathogenesis of leprosy. These factors participate both in the evolution of the clinical forms of leprosy and in the pathophysiology of reactional states, eg, Th9, Th17, Th22, and Th25. ${ }^{4}$

Among the components of an innate immune response, the inflammasome is a complex of cytosolic proteins that mediate the inflammatory response through pathogen-associated molecular patterns (PAMPs) and damage-associated molecular patterns (DAMPs), the latter being responsible for the maturation of caspase 1 , secretion of IL- $1 \beta$ and IL- 18 , and a type of cell death called pyroptosis. ${ }^{5-7}$ Inflammasomes are formed by pattern recognition receptors (PRRs), an adapter protein, and an effector enzyme (caspase). Depending on the type of the caspase involved, the inflammasomes can be classified into two types of signaling pathways: classical (ie, canonical), which activates caspase 1 , and noncanonical, which involves other caspases to provoke inflammation. ${ }^{7}$

Among the PRRs taking part in formation of the inflammasome, the nucleotide-binding and oligomerization domain (NOD)-like receptor (NLR) stands out. In humans, the NLR family of PRRs includes 22 types. Their basic structure consists of a variable $N$-terminal effector domain, a central NACHT domain, and the leucine repeat-rich $C$-terminal region. There are four NLR subfamilies classified according to their $N$ terminus: NLRA (acid activation domain), NLRB (BIR-type domain), NLRC (activation domain and caspase recruitment), and NLRP (pyrin domain). ${ }^{8,9}$

The accessory molecule called ASC (apoptosis-associated speck-like protein containing a Caspase activation and recruitment domains [CARD]) CARD (ASC) is an adapter protein common to several inflammasomes. It is formed by two protein domains, the N-terminal pyrin (PYD) and C-terminal caspase recruitment domain (CARD), and assists in formation of the inflammasome complex, creating a link between NLR and pro-caspase. ${ }^{10}$

Caspases are subdivided into two groups: inflammatory (caspases 1, 4, and 5) and apoptotic (initiators: caspases 2, 8, 9, and 10; executors: caspases 3, 6, and 7). Caspase- 1 is the key inflammatory caspase whose function is to activate IL-1 $\beta$ and IL-18, and activate the mediator of pyroptosis, gasdermin D. ${ }^{11}$ Other caspases may also be involved in the release of cytokines and mediating pyroptosis. Caspases 4 and 5 are activated by Gram-negative bacteria and may cleave gasdermin D, thereby contributing to pyroptosis. ${ }^{12,13}$ Caspase 8 can mediate inflammation and apoptosis, participating in formation of the inflammasome and activating IL-1 $\beta$, IL-18, and gasdermin $\mathrm{D}$. In addition, caspase 8 plays a part in the launch of the noncanonical pathway, which activates IL-1 $\beta$ independently of caspase 1 through dectin-1 receptor, a member of the lectin type $\mathrm{C}$ receptor family. ${ }^{13}$

The canonical inflammasome activation pathway is triggered when an NLR recognizes an intracellular PAMP or DAMP, thereby leading to recruitment of ASC into a PYDPYD binding complex. Then, pro-caspase 1 binds to ASC via a CARD-CARD connection. This action leads to canonical activation of the inflammasome, resulting in the production of IL-1 $\beta$ and IL-18 and in pyroptosis. ${ }^{14-16}$

The noncanonical inflammasome activation pathway can work in two ways. First, lipopolysaccharide (LPS) from Gramnegative bacteria activates caspases 4 and 5 , which then activates gasdermin D causing pyroptosis. LPS also switches on the nucleotide-binding oligomerization domain-like receptor containing pyrin domain 3 (NLRP3) inflammasome, which attracts caspase 1, resulting in the production of IL-1 $\beta$. The another noncanonical pathway involves the activation of caspase 8 by PAMPs recognized by lectin type $\mathrm{C}$ receptors. This event leads to the production of IL- $1 \beta$ independently from caspase $1 . .^{15,17}$

The role of inflammasomes has been addressed in some infectious diseases such as tuberculosis, leishmaniasis, ${ }^{18}$ paracoccidioidomycosis, ${ }^{19}$ and leprosy. ${ }^{20}$ Nonetheless, the interventions have mainly involved experimental models or in vitro studies. Triggering of NLRP3 has already been observed in the studies on Mycobacteria, eg, Mycobacteria tuberculosis $^{21}$ and Mycobacteria abscessus infections. ${ }^{22}$ In the case of M. tuberculosis, most of the studies have been based on in vitro experiments with macrophages and dendritic cells and revealed that NLRP3 is capable of inducing activation of the inflammasome. In addition, macrophages and M. tuberculosisinfected NLRP3-deficient dendritic cells show impaired IL-1 $\beta$ and IL-18 synthesis. Similar results have been observed with M. abscessus infection, where macrophages that do not express NLRP3 and ASC manifest reduced IL-1 $\beta$ production when infected. There are studies that have evaluated the participation of IL-1 $\beta$, IL-18, and caspase 1 in the inflammatory response in leprosy. ${ }^{23,24}$ Costa et al, ${ }^{24}$ who worked with infected and exposed individuals, have observed greater expression of IL-1 $\beta$ in the blood of patients undergoing treatment when compared to those exposed but not infected, indicating participation of this IL in the immunopathogenesis of the disease. Sinsimer et al, ${ }^{25}$ in their study on peripheral blood monocytes exposed to M. leprae, have detected a low IL-1 $\beta$ concentration 
and a high concentration of its natural inhibitor, IL-1Ra. In addition, these authors have reported reduced levels of IL-18, which were attributed to the delay in the activation of caspase 1. In mRNA studies on leprosy lesions, Garcia et $a^{23}$ have detected IL-18 in the majority of TT leprosy individuals and have not detected IL-18 in LL patients. Kang et $\mathrm{al}^{26}$ in a study on guinea pigs that have a defect in the synthesis of NAIP5, a protein of the NLR family, have verified the reduced activation of caspase 1 and IL- $1 \beta$ secretion, indicating participation of NLR proteins in the immunological response associated with leprosy. Pontillo et al have selected five single-nucleotide polymorphisms (SNPs) of nucleotide-binding oligomerization domain-like receptor containing pyrin domain 1 (NLRP1) and two SNPs of NLRP3 to study their possible influences on the development of leprosy. According to those authors, two combined NLRP1 haplotypes may be associated with a disease outcome, especially with paucibacillary forms. No associations of the NLRP3 polymorphisms with the disease were found. ${ }^{27}$ Given the complexity of the immune system, the importance of the in situ immune response against infectious conditions of the skin, and the relative absence of studies that characterize the expression of components of inflammasomes in cutaneous tissue, we decided to conduct this study. Here, we investigated the possible role of inflammasomes in the in situ immune response in skin lesions of leprosy. Specifically, we analyzed possible correlations of the immunological findings with the clinical forms of the disease. The results should contribute to a better understanding of the host immune response to $M$. leprae.

\section{Patients and methods Patients}

Forty-three untreated patients with a confirmed diagnosis of $M$. leprae infection were selected according to the criteria recommended by the Madrid classification (1953). All the patients sought the help of the dermatology service of the State University of Para between 2013 and 2017. Of the cases studied, 13 were suffering from I leprosy, 15 were suffering from TT leprosy, and 15 suffering from LL.

\section{Histopathological and immunohistochemical techniques}

Paraffin-embedded tissue biopsy sections ( $5 \mu \mathrm{m}$ thick) were stained with H\&E for histopathological analyses or with Ziehl-Neelsen acid-fast stain to detect Mycobacteria. Posterior tissue was stained with specific monoclonal antibodies for immunohistochemical analysis.
Immunohistochemical analysis with monoclonal antiNLRP1 (Abcam Cambridge, Cambridgeshire, UK; ab 98181), anti-NLRP3 (Abcam; ab 214185), anti-caspase 1 (Abcam; ab 18503), anti-IL-1 $\beta$ (Abcam; ab 14367), and anti-IL-18 (Abcam; ab 9722) antibodies followed the method involving formation of a biotin-streptavidin peroxidase complex according to the protocol described previously. ${ }^{28}$ Initially, tissue samples were dewaxed in xylene and dehydrated in ethyl alcohol. Then, endogenous peroxidases were blocked with $3 \% \mathrm{H}_{2} \mathrm{O}_{2}$ for 45 minutes. Antigen retrieval was performed in citrate buffer, $\mathrm{pH} 6$, at $95^{\circ} \mathrm{C}$ in a Pascal chamber for 20 minutes. Nonspecific binding sites were blocked with $10 \%$ concentrated skim milk for 30 minutes. The sections were then incubated with diluted primary antibodies (1:100) and $1 \%$ BSA for 14 hours. After that, the slides were washed with PBS and then incubated with the biotinylated secondary antibody (DakoCytomation, Glostrup, Denmark) for 30 minutes at $37^{\circ} \mathrm{C}$. The slides were again washed with PBS and incubated with a streptavidin-peroxidase conjugate (DakoCytomation) for 30 minutes at $37^{\circ} \mathrm{C}$. The slides were developed by application of the chromogen solution composed of $0.03 \%$ diaminobenzidine and $3 \% \mathrm{H}_{2} \mathrm{O}_{2}$, counterstained with Harris hematoxylin for 1 minute, then hydrated in ethyl alcohol, and diafiltered in xylene. Immunostaining results were quantified in five randomly selected visual fields under a Zeiss Axio Imager Z1 microscope (400×) by means of a graded lattice with $10 \times 10$ subdivisions at $0.0625 \mathrm{~mm}^{2}$.

\section{Statistical analyses}

The results obtained in the experiments were entered into Excel 2007 spreadsheets (Microsoft Corporation, Redmond, WA, USA). Statistical analyses were performed using GraphPad Prism 5.0 (GraphPad Software, Inc., La Jolla, CA, USA). In the univariate analysis, frequencies, measures of central tendency, and dispersion were obtained. Statistical comparisons involved ANOVA followed by Tukey's post hoc test or Pearson's correlation analysis. All the tests were performed considering a level of significance of $5 \%(P \leq 0.05)$.

\section{Ethics approval and consent to participate}

All the clinical analyses were conducted according to the principles expressed in the Declaration of Helsinki and Resolution No. 466/2012 of the National Health Council of Brazil. After a complete description and explanation of the study design, written informed consent was obtained from all the participants. The study protocol was approved by 
the ethics committee of Tropical Medicine Center, Federal University of Pará (protocol number: 1.827.246).

\section{Results}

\section{Clinical characteristics of the patients}

Twenty-three males and 20 females aged between 22 and 71 years from the eastern Brazilian Amazon, State of Pará, Brazil, were included in this study. Clinical features of the I leprosy were characterized by alterations of tactile, thermal, and/or pain sensitivity, with cutaneous lesions consisting of hypochromic spots with imprecise and sometimes hypoesthetic borders. Erythematous or erythematous and hypochromic plaques with sharp edges, usually anesthetic, were observed in TT leprosy group. Erythematous diffuse plaques and erythematous-violet nodules that were infiltrated, bright, and sometimes coalescing were observed in the LL group.

\section{Histopathological characteristics of the lesions}

As described by Eichelmann et al, ${ }^{1}$ histopathological features of lesions (Figure 1) in the I leprosy group included focal lymphocytic inflammatory infiltrates that were distributed around cutaneous appendages, nerve fibers, and vessels. In TT lesions, granulomas consisted of clustered epithelioid cells, sometimes surrounded by dense or mild lymphocytic halos without bacilli in the tissue. In LL lesions, the infiltrate

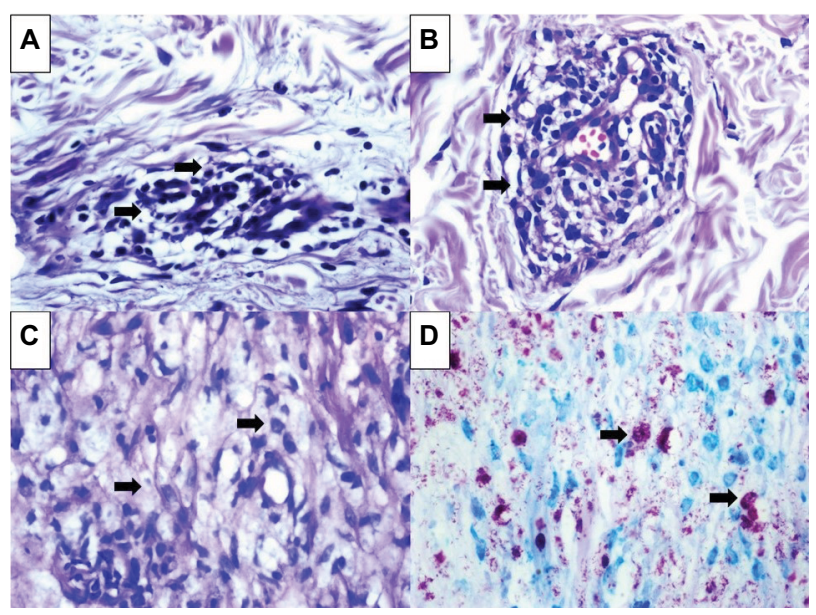

Figure I Histopathological features of leprosy lesions in I leprosy, TT leprosy, and LL showing focal lymphocytic inflammatory infiltrates that were distributed around cutaneous appendages, nerve fibers, and vessels in I leprosy.

Notes: TT leprosy shows granulomas consisting of clustered epithelioid cells, sometimes surrounded by lymphocytic halos. In LL, an inflammatory infiltrate consisting of histiocytes and plasma cells along the entire upper dermis was present around the nerves and blood vessels and around the bacillus stained by ZiehlNeelsen stain (200× magnification). (A) I leprosy (arrows indicate a lymphocytes), (B) TT leprosy (arrows indicate epithelial cells), (C) LL (arrows indicate histocytes), and (D) Ziehl-Neelsen staining (arrows indicate bacillus of $M$. leprae).

Abbreviations: I, indeterminate; LL, lepromatous leprosy; TT, tuberculoid. consisting of histiocytes and plasma cells that extended along the entire upper dermis was present around the nerves and blood vessels. These changes compromised the deep dermis until the hypodermis. Owing to the inability of macrophages to eliminate the bacilli, they accumulated in the cytoplasm, sometimes grouping together to form globes that were evident by Ziehl-Neelsen staining.

\section{Immunohistochemistry characteristics of the inflammatory skin infiltrate}

Immunostaining patterns of IL-1 $\beta$, IL-18, caspase 1, NLRP1, and NLRP 3 were characterized by a brownish color in the cytoplasm (Figure 2).

Quantification of the immunostaining for each factor under this study revealed statistically significant differences between I leprosy and TT leprosy and between I leprosy and LL. Expression of NLRP1 was the highest in lesions of LL (18.07 \pm 5.12 cells/field), followed by TT leprosy $(11.15 \pm 5.90$ cells/field) and I leprosy (8.28 \pm 5.11 cells/field), with the differences statistically significant between I leprosy and LL and between I leprosy and TT leprosy. There was no significant difference between the LL and TT leprosy groups (Figure 3 and Table 1). NLPR3 was expressed equally in the LL (15.67 \pm 3.55 cells/field) and TT (13.09 \pm 5.70 cells/field) leprosy groups. Expression in I leprosy $(8.46 \pm 4.51$ cells/ field) was significantly lower than that in LL and TT leprosy (Figure 3 and Table 1).

Caspase 1, a factor inducing pyroptosis, was most strongly expressed in LL (14.54 \pm 6.71 cells/field), followed by TT leprosy ( $6.41 \pm 7.56$ cells/field) and I leprosy $(7.73 \pm 5.78$ cells/field). There was a significant difference between the LL and I leprosy (Figure 3 and Table 1).

The expression of IL-1 $\beta$ and IL-18 was the highest in LL (IL-1 $\beta, 19.36 \pm 6.50$ cells/field; IL-18, 18.16 \pm 8.22 cells/ field), followed by TT leprosy (IL-1 $\beta, 13.24 \pm 9.38$ cells/field; IL-18, 13.13 \pm 5.11 cells/field) and I leprosy (IL-1 $\beta, 7.17 \pm 5.45$ cells/field; IL-18, 5.95 \pm 3.79 cells/field; Figure 3 and Table 1). Significant differences were observed between the LL and I leprosy in terms of IL-1 $\beta$ and IL-18. Significant differences were also observed between I leprosy and TT leprosy groups.

The correlation between the immunostaining intensity showed a positive and statistically significant correlation between the levels of caspase 1 and IL-18 in LL and TT leprosy between caspase 1 and IL-18 (LL: $r=0.7516, P=0.0012$; TT leprosy: $r=0.7366, P=0.0017$ ). Positive and statistically significant correlations were also found in I leprosy form between IL-1 $\beta$ and caspase $1(r=0.6412, P=0.0182)$, between IL-18 and NLRP1 ( $r=0.5585, P=0.0473)$, between IL-18 and 


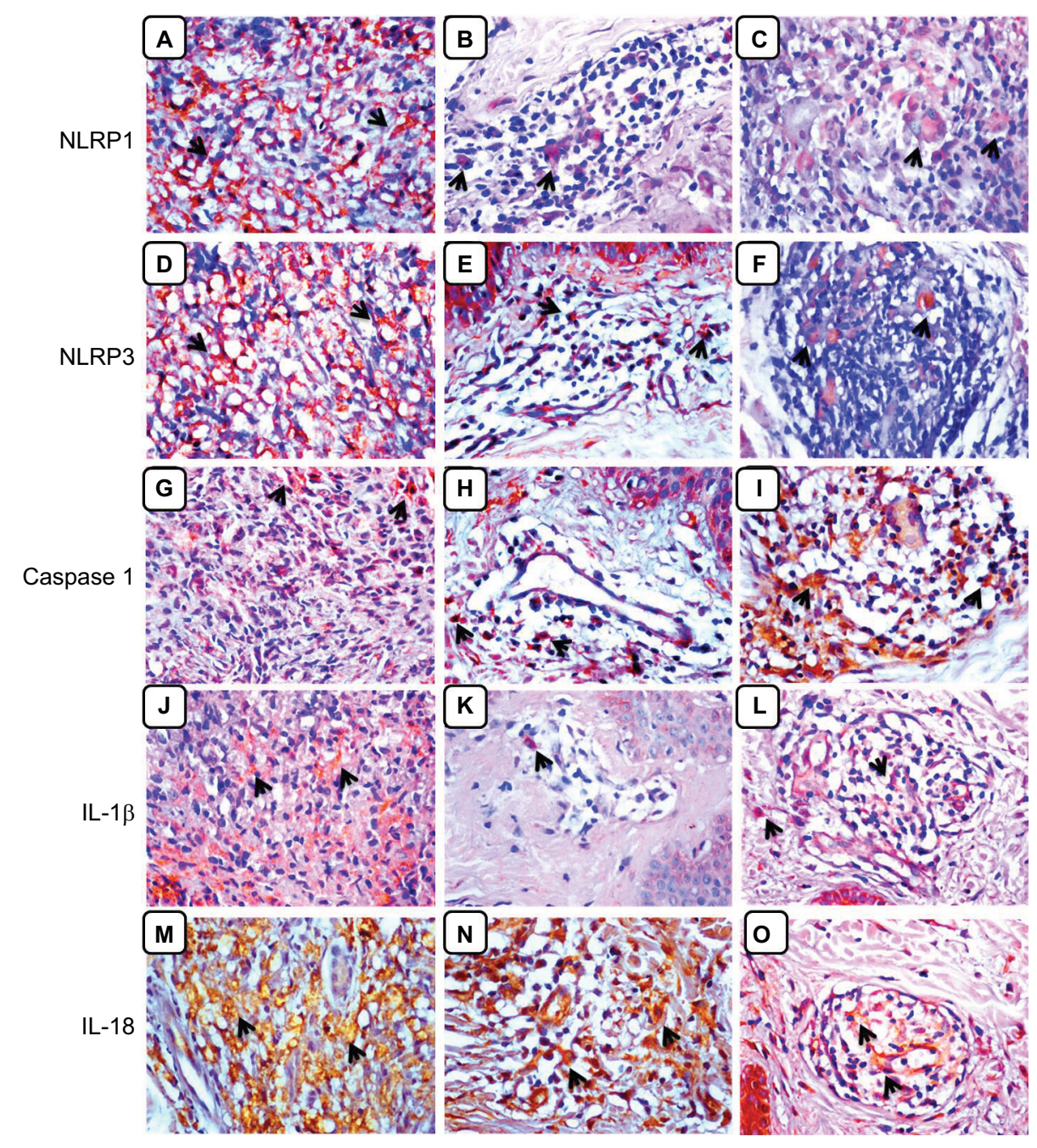

Figure 2 Immunohistochemical patterns of the inflammasome in leprosy skin lesions.

Notes: Note that immunostaining occurs predominantly in immune cells of the granulomatous inflammatory infiltrate representative of the clinical forms LL, I leprosy, and TT leprosy. Immunostaining for NLRPI in LL (A), I leprosy (B), and TT leprosy (C); for NLRP3 in LL (D), I leprosy (E), and TT leprosy (F); for caspase I in LL (G), I leprosy $(\mathbf{H})$, and TT leprosy $(\mathbf{I})$; for IL-I $\beta$ in LL (J), I leprosy $(\mathbf{K})$, and TT leprosy $(\mathbf{L})$; and for IL-I 8 in LL (M), I leprosy $(\mathbf{N})$, and TT leprosy $(\mathbf{O})$. The arrows indicate the stained areas bounded by brownish color coincident with the cell bodies in the granulomatous inflammatory infiltrate (magnification, 200X).

Abbreviations: I, indeterminate; LL, lepromatous leprosy; NLRPI, nucleotide-binding oligomerization domain-like receptor containing pyrin domain I; NLRP3, nucleotidebinding oligomerization domain-like receptor containing pyrin domain 3; TT, tuberculoid.

NLRP3 $(r=0.6873, P=0.0094)$, and between NLRP1 and NLRP3 ( $r=0.8040, P=0.0009$; Figure 4).

\section{Discussion}

Leprosy is a chronic disease that causes several changes in host defense systems. ${ }^{29}$ In the context of studying various effects of the immune system against infectious agents, the importance of identifying systems with characteristics and properties adapted to each organ has been revealed in the study by Engwerda and Kaye. ${ }^{30}$ Thus, according to the study by Quaresma et al, ${ }^{31}$ we can subdivide the patterns of tissue responses into three major groups: barrier immunity, complex organ immunity, and immunologically privileged organ immunity. The skin has immune characteristics that make it a complex organ. Furthermore, the skin manifests peculiar characteristics in its interaction with various infectious agents. In particular, sometimes the skin can be considered an organ with its own immune system that often employs different mechanisms when responding to infections compared to other organs of the body. ${ }^{32}$

Among the mechanisms triggered by innate immunity in response to infectious agents, inflammasomes are complexes of cytoplasmic molecules (proteins) that are induced in response to PAMPS and DAMPS in the contexts of infection and cellular stress. ${ }^{5}$ Inflammasomes can be activated by microbial components, such as flagellin, LPS, pore-forming toxins, bacterial and viral RNAs, and endogenous and environmental crystals, and by reduced intracellular potassium 
A

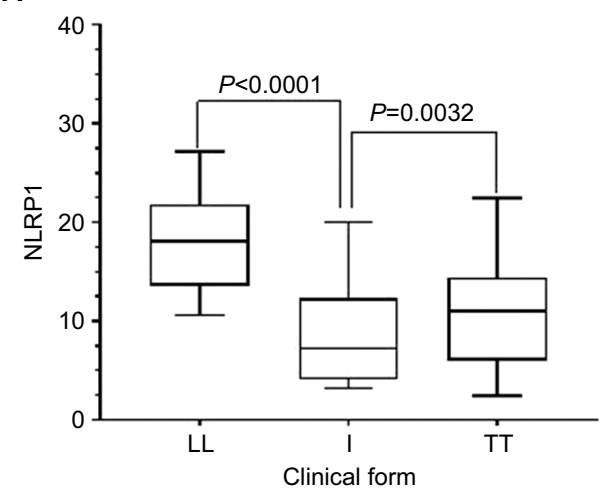

C

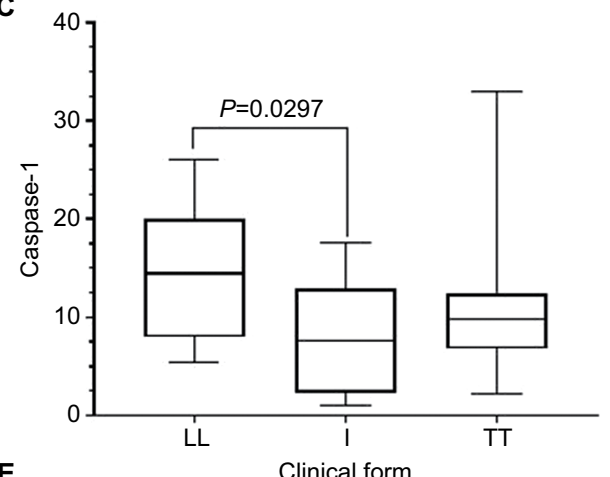

E

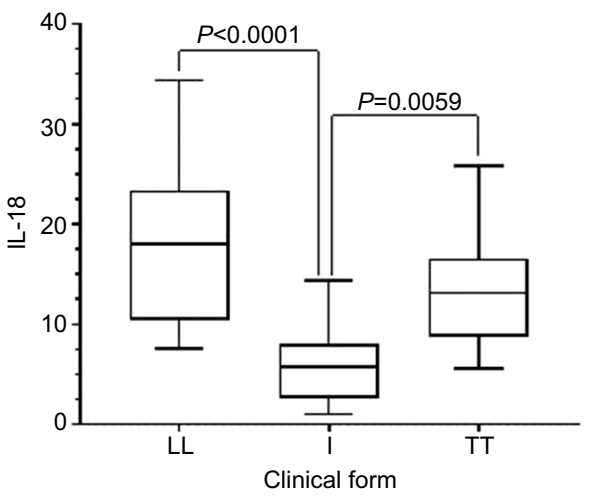

B

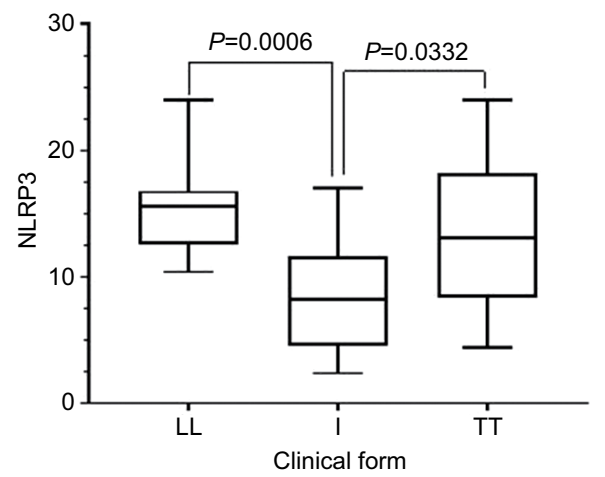

D

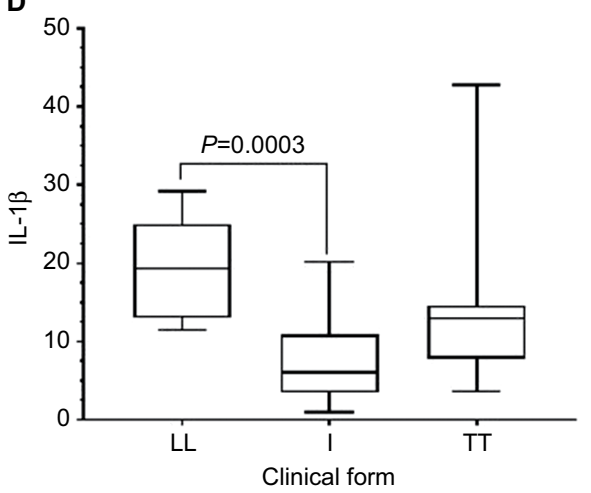

Figure 3 Comparative analysis of the means and SDs of NLRPI, NLRP3, caspase I, IL-I $\beta$, and IL-I8 expression by leprosy clinical form.

Notes: Comparative analysis of the means and SDs of NLRPI (A), NLRP3 (B), Caspase-I (C), IL-I $\beta$ (D), and IL-I8 (E) expression by leprosy clinical form. Note the increased expression of the proteins investigated by immunohistochemistry in cutaneous lesions of LL when compared with I leprosy and TT leprosy. Statistically significant differences were observed between the clinical forms I leprosy and TT leprosy and between I leprosy and LL in the levels of markers NLRPI, NLRP3, and IL-I8. As for caspase I and IL-I $\beta$, significant differences were observed between I leprosy and LL.

Abbreviations: I, indeterminate; LL, lepromatous leprosy; NLRPI, nucleotide-binding oligomerization domain-like receptor containing pyrin domain I; NLRP3, nucleotidebinding oligomerization domain-like receptor containing pyrin domain 3; TT, tuberculoid.

Table I The mean and SD of the immunostaining data on the inflammasome proteins in the skin lesions of the clinical forms of leprosy

\begin{tabular}{|c|c|c|c|c|}
\hline \multirow[t]{2}{*}{ Markers } & \multicolumn{3}{|l|}{ Clinical forms of leprosy } & \multirow[t]{2}{*}{$P$-value ${ }^{a}$} \\
\hline & $\begin{array}{l}\text { LL, } \\
\text { mean } \pm S D(95 \% \mathrm{Cl})\end{array}$ & $\begin{array}{l}\text { I leprosy, } \\
\text { mean } \pm \text { SD (95\% Cl) }\end{array}$ & $\begin{array}{l}\text { TT leprosy, } \\
\text { mean } \pm \text { SD }(95 \% \mathrm{Cl})\end{array}$ & \\
\hline NRLPI & $18.07 \pm 5.12(15.24-20.91)$ & $8.28 \pm 5.11(5.19-11.36)$ & $11.15 \pm 5.90(7.88-14.42)$ & $<0.000 I^{b}$ \\
\hline NLRP3 & $15.67 \pm 3.55(13.70-17.63)$ & $8.46 \pm 4.5 \mathrm{I}(5.74-\mathrm{II} .19)$ & 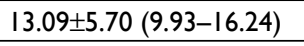 & $0.0009^{b}$ \\
\hline Caspase-I & $|4.54 \pm 6.7|(|0.83-| 8.25)$ & $7.73 \pm 5.78$ (4.24-II.22) & $6.4 I \pm 7.56(6.4 I-\mid 4.78)$ & $0.0367^{\mathrm{b}}$ \\
\hline IL-I $\beta$ & $19.36 \pm 6.50(15.76-22.96)$ & $7.17 \pm 5.45(3.87-10.42)$ & |3.24士9.38 (8.05-|8.44) & $0.0004^{\mathrm{b}}$ \\
\hline IL-18 & $18.16 \pm 8.22(|3.6|-22.7 \mid)$ & $5.95 \pm 3.79(3.66-8.23)$ & $13.13 \pm 5.1 \mid(10.30-15.96)$ & $<0.000 \mathrm{I}^{\mathrm{b}}$ \\
\hline
\end{tabular}

Notes: More intense expression of the various proteins being analyzed was observed in LL when compared to clinical forms I leprosy and TT leprosy. ${ }^{2}$ Ordinary one-way ANOVA $(P<0.05)$. 'Statistically significant.

Abbreviations: I, indeterminate; LL, lepromatous leprosy; NLRPI, nucleotide-binding oligomerization domain-like receptor containing pyrin domain I; NLRP3, nucleotidebinding oligomerization domain-like receptor containing pyrin domain 3; TT, tuberculoid. 
A

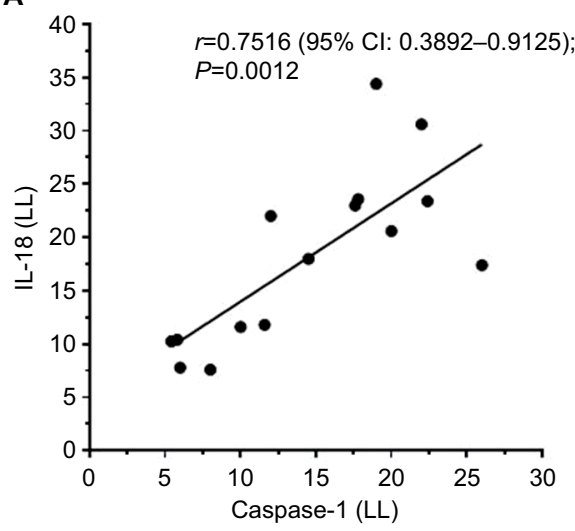

C

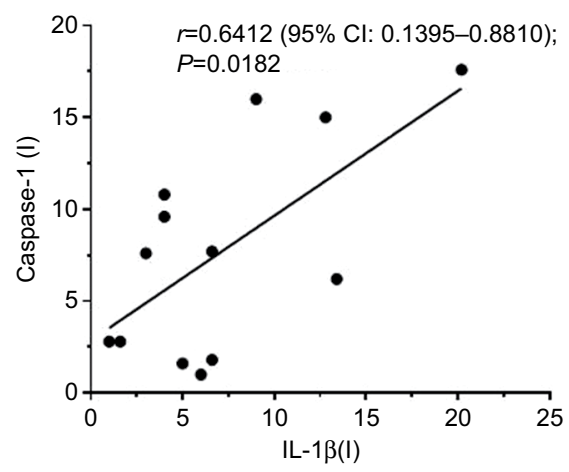

E

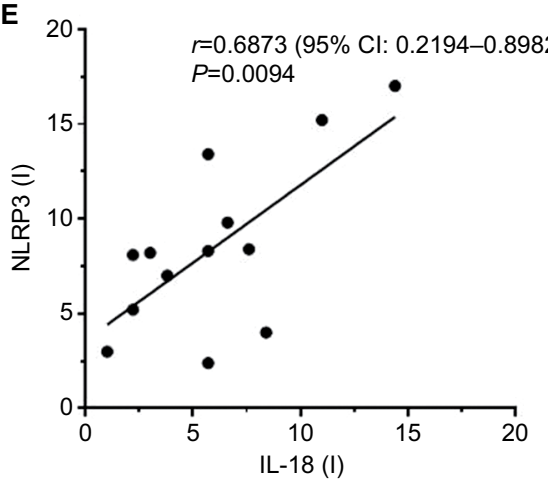

B
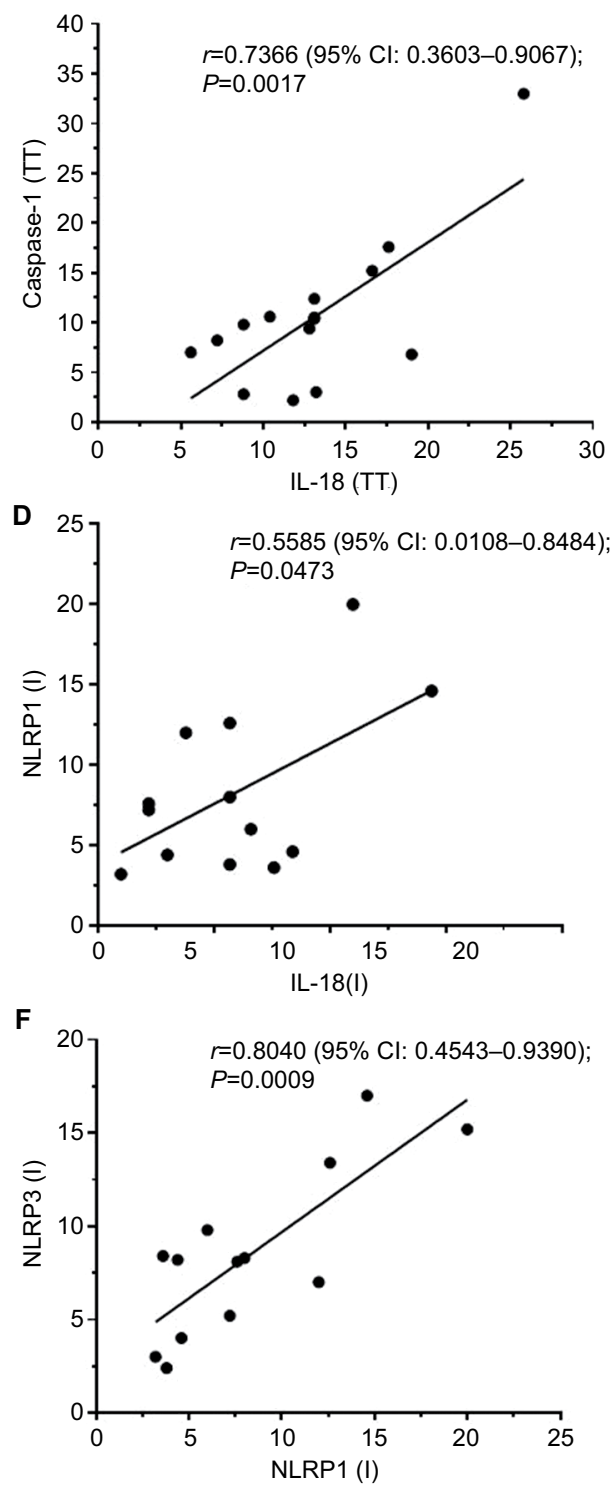

Figure 4 Statistically significant correlations between IL-I8 and caspase I in LL (A) and TT leprosy (B), between caspase I and IL-I $\beta$ (C), between NLRPI and IL-I8 (D), between NLRP3 and IL-I8 (E), and between NLRP3 and NLRPI (F) in I leprosy.

Abbreviations: I, indeterminate; LL, lepromatous leprosy; NLRPI, nucleotide-binding oligomerization domain-like receptor containing Pyrin domain I; NLRP3, nucleotidebinding oligomerization domain-like receptor containing pyrin domain 3; TT, tuberculoid.

concentration. There is evidence that NLRPs are not activated directly by damaging agents but rather by cytoplasmic changes that lead to a reduction in the intracellular potassium concentration consequent to pore-forming toxins, cellular stress, or the production of ROS that can induce cellular injury. ${ }^{16}$ Stimulation of caspases resulting from NLRP activation can lead to pyroptosis and a release of inflammatory mediators such as IL-1 $\beta .^{12}$

Dectin-1 is a type of C-lectin recognition receptor present primarily in dendritic cells and is targeted during induction of a Th1 or Th17 protective response. ${ }^{33}$ According to two studies, ${ }^{20,34}$ dectin-1 acts directly as a sensor for extracellular
PAMPs of Mycobacteria and activates caspase 8-driven processing of pro-IL-1 $\beta$. The production of IL- $1 \beta$ in response to the stimulation of dendritic cells with $M$. leprae proceeds primarily via dectin- 1 , and this secretion of IL- $1 \beta$ is completely dependent on caspase $8 .^{29}$ Thus, recognition of $M$. leprae by dectin-1 causes noncanonical inflammasome-dependent activation of caspase 8 and processing of pro-IL-1 $\beta .^{29}$

The binding of dectin-1 to pathogens triggers formation and activation of a noncanonical inflammasome complex consisting of CARD9, Bcl-10, MALT1, caspase 8, and ASC; this complex processes pro-IL- $1 \beta$ into its biologically active form. Intracellular PRRs, such as NLR proteins, recognize 
intracellular pathogens, whereas dectin-1 detects PAMPs from $M$. leprae, thus allowing for a quick response to the pathogen without requiring internalization. ${ }^{20}$ The existence of a caspase-8-dependent noncanonical inflammasome highlights the diversity and versatility of immune responses to infection. In our samples of lesions, an increase in inflammasome expression among the clinical forms of leprosy under study indicates that the canonical pathway of inflammasome activation also participates in response to $M$. leprae in the skin..$^{20,29}$ Nevertheless, the predominant expression of inflammasomes in LL lesions also shows their relative lack of efficacy in the control of infection in the skin.

Several studies have evaluated the involvement of IL-1 $\beta$, IL-18, and caspase 1 in the inflammatory response to leprosy. ${ }^{35,36}$ Garcia et al ${ }^{35}$ have analyzed individuals exposed to (and infected with) M. leprae and observed greater expression of IL-1 $\beta$ in the blood of patients undergoing treatment when compared to those exposed but not infected, indicating participation of IL-1 $\beta$ in the immunopathogenesis of the disease. In a study on peripheral blood monocytes exposed to $M$. leprae ${ }^{24}$ researchers detected a low level of IL- $1 \beta$ and a large amount of its natural inhibitor, IL-1Ra. In addition, the authors also reported reduced levels of IL-18, which were attributed to delayed activation of caspase 1 . In our patients, an increased expression of caspase 1 in LL skin lesions may be related to an increase in cell death by pyroptosis. This is especially true in infected macrophages that are incapable of eliminating the bacillus, where caspase 1 may be induced by components of $M$. leprae or the consequent release of reactive oxygen or nitrogen species by activated macrophages leading to stress and cell injury with efflux of potassium. ${ }^{25}$

Some studies have shown that both necrosis and apoptosis are important mechanisms of cell death during mycobacterial infections, including $M$. tuberculosis and M. leprae. Our results indicate that pyroptosis can constitute another mechanism of cell death in the course of leprosy. Pyroptosis may be related to an underlying tissue injury mechanism of the disease that inhibits the response of macrophages. In addition, studies by Elamin et $\mathrm{al}^{36}$ and Mattos et $\mathrm{al}^{37}$ have revealed the mechanism by which lipid-filled foam cells emerge in skin lesions in anergic leishmaniasis and LL. This mechanism seems to be directly related to the greater expression of inflammasome components in these lesions that then deposit various substances including cholesterol, free fatty acids, and lipids.

In studies assessing the patterns of mRNA expression in leprosy lesions, ${ }^{28}$ expression of IL-18 has been detected in most patients with TT leprosy but not in patients with LL. Nonetheless, it is noteworthy that mRNA expression does not always correlate with protein synthesis. The in situ immune response evaluated in our samples was measured in terms of protein expression, which may partially explain the aforementioned discrepancy. ${ }^{36-38}$

A study on mice with defects in the synthesis of NAIP5, ${ }^{39,40}$ a member of the NLR family, has shown reduced caspase 1 and IL-1 $\beta$ levels, indicating the involvement of NLR proteins in the immune response to leprosy. This result was corroborated by the expression of NLRP1 and NLRP3 in our samples of the three clinical forms of leprosy.

In a study on polymorphisms, ${ }^{39}$ five SNPs of NLRP1e and two SNPs of NLRP3 were found to potentially influence the development of leprosy. The authors suggested that two combined NLRP1 haplotypes may be associated with a disease outcome, especially with TT leprosy. No associations of the NLRP3 polymorphism with the disease were found. Our data uncovered the activation of both NLRP1 and NLRP3 in leprosy and both were more highly expressed in LL when compared to I leprosy and TT leprosy.

NLRP1 and NLRP3 are expressed in various cellular populations involved in the immune response to $M$. leprae, including macrophages, dendritic cells, lymphocytes, and granulocytes, and therefore actively participate in the immune response to leprosy. ${ }^{39-42}$

NLRP1 is the first NLR protein that has been shown to form a cytosolic complex called the inflammasome, whose functions include specific recruitment and activation of a protease called caspase 1 . Both NLRP1 and caspase 1 were found here to be more expressed in LL and less expressed in TT leprosy; these data indirectly support a correlation between the activation of NLRP1 and caspase 1 in the immunopathogenesis of this disease. ${ }^{39-42}$

In addition, research has shown that activated NLRP3 leads to the release of activated caspase 1 , leading to maturation and secretion of IL-1 $\beta$ and IL-18. Our results revealed statistically significant positive linear correlations among caspase 1 , IL-1 $\beta$, and IL-18. These data are confirmed by the increased expression of caspase 1 , IL-1 $\beta$, and IL-18 in LL and the decreased expression of caspase 1, IL-1 $\beta$, and IL-18 in I leprosy and TT leprosy. The process of activation of NLRP3 is mediated by at least two types of signals: 1) activation of a toll-like receptor through expression of NLRP3, pro-IL-1 $\beta$, and pro-IL-18 and 2) a signal that leads to the autocatalytic activation of pro-caspase 1 with proteolytic cleavage of pro-IL- $1 \beta$ and pro-IL-18. ${ }^{39-42}$

Our findings point to the major participation of NLRP1 and NLRP3 and their associations with the activation of caspase 1 , IL-1 $\beta$, and IL-18 during the immune response to M. leprae. Nevertheless, additional research is needed 
to complement the findings of the present study, especially research based on molecular biological methods and protein analysis techniques to better characterize the role of inflammasomes in the pathogenesis of leprosy.

\section{Conclusion}

Regardless of the experimental model used, our results are suggestive of effective participation of an inflammasome-mediated response in the clinical evolution of leprosy. This participation begins with the first contact with the bacillus at the early stages of the disease. The outcome of this initial interaction is important for the clinical course of the infection, and it appears that at this stage, NLRP1, NLRP3, caspase 1, IL-1 $\beta$, and IL-18 cooperate in an integrated manner to elicit the initial response. This notion is supported by the correlation analyses that are more significant in I leprosy than in LL and TT leprosy. This initial interaction of the pathogen with components of the host's innate immunity contribute decisively to the development of a more or less effective immune response to $M$. leprae infection and seems to be a crucial step (in the entire natural course of the disease) in terms of host-pathogen interactions in leprosy.

\section{Data sharing statement}

The data sets used and/or analyzed during this study are available from the corresponding author on reasonable request.

\section{Acknowledgments}

We thank the Pará State University Dermatology Service for supporting the development of this study. Dr Juarez AS Quaresma is a Research Productivity Fellow and Senior Postdoctoral Fellow at the Brazilian National Council for Scientific and Technological Development - CNPq/Brazil (grants numbers 302553/2015-0 and 116427/2016-7). Program of support for qualified production-PAPQ/UFPA.

\section{Author contributions}

LMS, JRdS, KEH, MNS, and JASQ contributed to research design; LMS, KEH, LBDJ, and FROC were involved in the acquisition of data; and IPF, TLdSA, JRdS, MNS, and JASQ participated in analysis and interpretation of the data. All the coauthors were involved in drafting the manuscript and/ or revising it critically, and all the coauthors approved the submitted final version. All authors contributed toward data analysis, drafting and revising the paper, gave final approval of the version to be published, and agree to be accountable for all aspects of the work.

\section{Disclosure}

The authors report no conflicts of interest in this work.

\section{References}

1. Eichelmann K, González González SE, Salas-Alanis JC, OcampoCandiani J. Leprosy. An update: Definition, pathogenesis, classification, diagnosis, and treatment. Acta Dermosifiliogr. 2013;104(7):554-563.

2. Saini C, Tarique M, Rai R, Siddiqui A, Khanna N, Sharma A. T helper cells in leprosy: An update. Immunol Lett. 2017;184:61-66.

3. de Alvarenga Lira ML, Pagliari C, de Lima Silva AA, de Andrade HF Jr, Duarte MI. Dermal dendrocytes FXIIIa+ are essential antigenpresenting cells in indeterminate leprosy. Am J Dermatopathol. 2015;37(4):269-273.

4. de Sousa JR, Sotto MN, Simões Quaresma JA. Leprosy As a Complex Infection: Breakdown of the Th1 and Th2 Immune Paradigm in the Immunopathogenesis of the Disease. Front Immunol. 2017;8:1635.

5. Amin J, Boche D, Rakic S. What do we know about the inflammasome in humans? Brain Pathol. 2017;27(2):192-204.

6. Zamboni DS, Lima-Junior DS. Inflammasomes in host response to protozoan parasites. Immunol Rev. 2015;265(1):156-171.

7. Broz P, Dixit VM. Inflammasomes: mechanism of assembly, regulation and signalling. Nat Rev Immunol. 2016;16(7):407-420.

8. Lu A, Wu H. Structural mechanisms of inflammasome assembly. FEBS J. 2015;282(3):435-444

9. Garib FY, Rizopulu AP, Kuchmiy AA, Garib VF. Inactivation of inflammasomes by pathogens regulates inflammation. Biochemistry. 2016;81(11):1326-1339.

10. Sharma D, Kanneganti TD. The cell biology of inflammasomes: Mechanisms of inflammasome activation and regulation. $J$ Cell Biol. 2016;213(6):617-629.

11. Uchiyama R, Tsutsui H. Caspases as the key effectors of inflammatory responses against bacterial infection. Arch Immunol Ther Exp. 2015;63(1):1-13.

12. Jiménez Fernández D, Lamkanfi M. Inflammatory caspases: key regulators of inflammation and cell death. Biol Chem. 2015;396(3):193-203.

13. Man SM, Karki R, Kanneganti TD. Molecular mechanisms and functions of pyroptosis, inflammatory caspases and inflammasomes in infectious diseases. Immunol Rev. 2017;277(1):61-75.

14. Lechtenberg BC, Mace PD, Riedl SJ. Structural mechanisms in NLR inflammasome signaling. Curr Opin Struct Biol. 2014;29:17-25.

15. Vanaja SK, Rathinam VA, Fitzgerald KA. Mechanisms of inflammasome activation: recent advances and novel insights. Trends Cell Biol. 2015;25(5):308-315.

16. Kesavardhana S, Kanneganti TD. Mechanisms governing inflammasome activation, assembly and pyroptosis induction. Int Immunol. 2017;29(5):201-210.

17. Lamkanfi M, Dixit VM. A new lead to NLRP3 inhibition. J Exp Med. 2017;214(11):3147-3149.

18. Lima-Junior DS, Costa DL, Carregaro V, et al. Inflammasome-derived IL-1 $\beta$ production induces nitric oxide-mediated resistance to Leishmania. Nat Med. 2013;19(7):909-915.

19. Tavares AH, Magalhães KG, Almeida RD, Correa R, Burgel PH, Bocca AL. NLRP3 inflammasome activation by Paracoccidioides brasiliensis. PLoS Negl Trop Dis. 2013;7(12):e2595.

20. Gringhuis SI, Kaptein TM, Wevers BA, et al. Dectin-1 is an extracellular pathogen sensor for the induction and processing of IL- $1 \beta$ via a noncanonical caspase-8 inflammasome. Nat Immunol. 2012;13(3):246-254.

21. Briken V, Ahlbrand SE, Shah S. Mycobacterium tuberculosis and the host cell inflammasome: a complex relationship. Front Cell Infect Microbiol. 2013;3:62.

22. Lee HM, Yuk JM, Kim KH, et al. Mycobacterium abscessus activates the NLRP3 inflammasome via Dectin-1-Syk and p62/SQSTM1. Immunol Cell Biol. 2012;90(6):601-610.

23. Garcia VE, Uyemura K, Sieling PA, et al. IL-18 promotes type 1 cytokine production from NK cells and T cells in human intracellular infection. J Immunol. 1999;162(10):6114-6121.

24. Costa RD, Mendonça VA, Lyon S, et al. Evaluation of the expression of interleukin 1 beta (IL-1beta) and interleukin 1 receptor antagonist (IL-1Ra) in leprosy patients. Rev Soc Bras Med Trop. 2008;41 (Suppl 2):99-103. 
25. Sinsimer D, Fallows D, Peixoto B, et al. Mycobacterium leprae actively modulates the cytokine response in naive human monocytes. Infect Immun. 2010;78(1):293-300.

26. Kang TJ, Lee GS, Kim SK, Jin SH, Chae GT. Comparison of two mice strains, A/J and C57BL/6, in caspase-1 activity and IL- $1 \beta$ secretion of macrophage to Mycobacterium leprae infection. Mediators Inflamm. 2010;2010:708713.

27. Pontillo A, Laurentino W, Crovella S, Pereira AC. NLRP1 haplotypes associated with leprosy in Brazilian patients. Infect Genet Evol. 2013;19: 274-279.

28. Quaresma JA, Barros VL, Pagliari C, et al. Revisiting the liver in human yellow fever: virus-induced apoptosis in hepatocytes associated with TGF-beta, TNF-alpha and NK cells activity. Virology. 2006;345(1): 22-30.

29. Cardoso CC, Pereira AC, de Sales Marques C, Moraes MO. Leprosy susceptibility: genetic variations regulate innate and adaptive immunity, and disease outcome. Future Microbiol. 2011;6(5):533-549.

30. Engwerda CR, Kaye PM. Organ-specific immune responses associated with infectious disease. Immunol Today. 2000;21(2):73-78.

31. Quaresma JAS, Sotto MN, Balato A. Inflammatory and ImmuneMediated Cutaneous Diseases. Mediators Inflamm. 2017;2017:6793968.

32. Tay SS, Roediger B, Tong PL, Tikoo S, Weninger W. The Skin-Resident Immune Network. Curr Dermatol Rep. 2014;3:13-22.

33. Duluc D, Joo H, Ni L, et al. Induction and activation of human Th17 by targeting antigens to dendritic cells via dectin-1. J Immunol. 2014;192(12):5776-5788.
34. Lee HM, Yuk JM, Kim KH, et al. Mycobacterium abscessus activates the NLRP3 inflammasome via Dectin-1-Syk and p62/SQSTM1. Immunol Cell Biol. 2012;90(6):601-610.

35. García VE, Uyemura K, Sieling PA, et al. IL-18 promotes type 1 cytokine production from NK cells and T cells in human intracellular infection. J Immunol. 1999;162(10):6114-6121.

36. Elamin AA, Stehr M, Singh M. Lipid Droplets and Mycobacterium leprae Infection. J Pathog. 2012;2012:361374.

37. Mattos KA, Lara FA, Oliveira VG, et al. Modulation of lipid droplets by Mycobacterium leprae in Schwann cells: a putative mechanism for host lipid acquisition and bacterial survival in phagosomes. Cell Microbiol. 2011;13(2):259-273.

38. Kang TJ, Lee GS, Kim SK, Jin SH, Chae GT. Comparison of two mice strains, A/J and C57BL/6, in caspase-1 activity and IL-1beta secretion of macrophage to Mycobacterium leprae infection. Mediators Inflamm. 2010;2010:708713.

39. Pontillo A, Laurentino W, Crovella S, Pereira AC. NLRP1 haplotypes associated with leprosy in Brazilian patients. Infect Genet Evol. 2013;19: 274-279.

40. Chavarría-Smith J, Vance RE. The NLRP1 inflammasomes. Immunol Rev. 2015;265(1):22-34.

41. Awad F, Assrawi E, Louvrier C, et al. Inflammasome biology, molecular pathology and therapeutic implications. Pharmacol Ther. 2018;187:133-149.

42. Evavold CL, Kagan JC. How Inflammasomes Inform Adaptive Immunity. J Mol Biol. 2018;430(2):217-237.
Infection and Drug Resistance

\section{Publish your work in this journal}

Infection and Drug Resistance is an international, peer-reviewed openaccess journal that focuses on the optimal treatment of infection (bacterial, fungal and viral) and the development and institution of preventive strategies to minimize the development and spread of resistance. The journal is specifically concerned with the epidemiology of antibiotic
Dovepress

resistance and the mechanisms of resistance development and diffusion in both hospitals and the community. The manuscript management system is completely online and includes a very quick and fair peerreview system, which is all easy to use. Visit http://www.dovepress.com/ testimonials.php to read real quotes from published authors. 